\title{
Stochastic Variation of the Hubble's Parameter using Ornstein- Uhlenbeck Process
}

\author{
Mary Chriselda A.
}

\begin{abstract}
The premise of this paper proves that the constant value of the Hubble's parameter tends to vary stochastically with time. By the use of Ornstein-Uhlenbeck process a stochastic differential equation has been formulated and solved using the Euler and the Kolmogorov Forward equations. We can further draw conclusion that the randomness in forces affecting the white noise can eventually affect the Hubble Parameter's behavior leading to scale invariance and thereby causing stochastic fluctuations in the density and the rate of expansion of the Universe. This is further validated by simulating the observations using Python and R-software for validation of the premise postulated.[17]
\end{abstract}

\section{General Terms}

Astrophysics, Mathematical Modelling and Simulations.

\section{Keywords}

Hubble's Parameter, Chapman Kolmogorov Forward Differential Equations, Stochastic Differential Equations, Ornstein-Uhlenbeck process, Fourier Transformation

\section{INTRODUCTION}

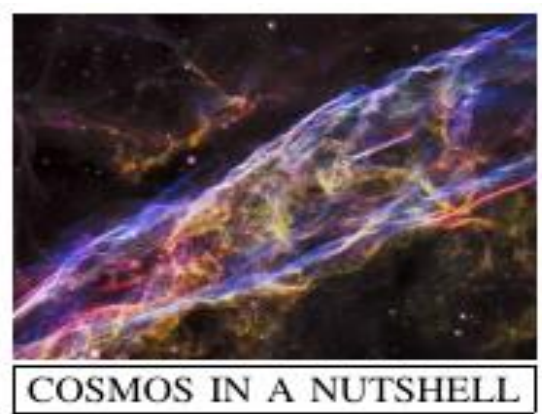

Fig. 1: SOURCE : HUBBLE TELESCOPE

$\mathrm{E}^{2}$ dwin Hubble introduced the Hubble's Law, which gives the association of the distance and radial velocities of the galaxies. The Hubble's Law talks of the rate of expansion of the Universe. All standard cosmological models based on the two fundamental principles mentioned below follows the Hubble's Law.

1. The Universe is homogenous.

2. It is isotropic in nature.

Hubble's Law explains the rate at which the galaxies are receding is proportional to the distance covered which uses the observations to determine the proportionality constant which is famously called the Hubble's constant.

But this assertion was filled with lacunae as a matter of fact Hubble's distances to the nearby local galaxies were too small. These results were further proven erroneous as the caveats like "extrapolation", "hypothesis", "assumptions" were extensively used implying that it was a conjecture that further needed to be substantiated.

$v=H_{0} r$

This paper has been organized into the following sections:

- Section 1 deals with the Introduction followed by the Literature Survey.

- Section 2 gives the proof for the Formulation of the Robertson-Walker Metric Equation,2.2 deals with Friedmann Equation.

- Section 3 is about the Stochastic Differential Equation Using Ornstein-Uhlenbeck Process

- Section 4 deals with the Analytical Methods for Solving SDE where 4.1 is about the EulerMaruyama Method and section 4.2 is about the Chapman Kolmogorov Forward Differential Equations

- Section 5 deals with the Results Analysis divided as Sensitivity Analysis and Simulation Results

- Section 6 is Conclusion followed by Section 7 which is Acknowledgement and finally concluded with the References Section.

\subsection{Literature Survey}

[1]Around 1917 Vesto Sipher observed around 25 galaxies spreading their light out with the help of a prism and recorded these results wherein he found the light expanding to redder hues .This definitely gave out a strong signal that the Universe was moving away us .In 1927 Belgian monk Georges Lemaitre derived the Friedmann cosmological solutions to general relativity .Around the same time in 1928 Robertson at Caltech predicted the Hubble's Law, reflecting the nature of the expansion of the Universe.

Edwin Hubble collected the galaxy distances known at that time which were based on the observations of Cepheid variable stars to calibrate these absolute magnitudes of the galaxies. The data of the sample has been provided in the Results Section.

Many recent research undertaken like the [11] cFA,Las Campanas,SSRS,LEDA,IRAS, ESP ,Abell and ACO have had myriad revelations on the three-dimensional distribution of galaxies and cluster characterized by the huge hierarchical voids and galactic fractals.

\section{ROBERTSON WALKER METRIC}

With Einstein's general relativity concept, that matter could control the metric stating how far apart two elements gave a lot of convenience as to what the metric can be or not which was discovered by Alexander Friedmann, because of the two fundamental principles of cosmology. We have considered here the spherical coordinates which are the same coordinates used to measure the position on the sky. This metric describes the central notion of distance with the three geometrics flat, 
closed and open universe.

Definition:

The definition of a metric in three-dimensional space can be expressed as distance along a curvilinear path say $\mathrm{P}$ in between two points $\mathrm{A}$ and $\mathrm{B}$ using the differential distance formula or metric:

Proof:

$(\mathrm{dl})^{2}=(\mathrm{dx})^{2}+(\mathrm{dy})^{2}+(\mathrm{dz})^{2}$

We integrate along this curvilinear path say $\mathrm{P}$ to get the total distance

$\delta \mathrm{l}=\int_{1}^{2} \sqrt{(\mathrm{dl})^{2}}=\int_{1}^{2} \sqrt{\left\{(\mathrm{dx})^{2}+(\mathrm{dy})^{2}+(\mathrm{dz})^{2}\right\}}$

Now to connect two events in space time with no mass we use the metric for a flat universe

$\mathrm{ds}^{2}=\mathrm{cdt}^{2}-\mathrm{dx} \mathrm{x}^{2}-\mathrm{dy}^{2}-\mathrm{dz}^{2}$

Integrating to get the total interval

$\delta s=\int_{\mathrm{A}}^{\mathrm{B}} \sqrt{(\mathrm{ds})^{2}}=\int_{\mathrm{A}}^{\mathrm{B}} \sqrt{\left\{\mathrm{cdt}^{2}-\mathrm{dx}^{2}-\mathrm{dy}^{2}-\mathrm{dz}^{2}\right\}}$

Thus, we have found the metric that describes the space time of a matter filled universe by the two fundamental principles of cosmology. Now we can describe the curvature on a sphere

$\mathrm{k}=\frac{1}{\mathrm{R}^{2}}$

We know that the circumference of a circle is equal to radius $2 \prod$ only in Euclidean geometry. The distance between any two points on the surface of the sphere is given by

$(\mathrm{dl})^{2}=(\mathrm{dD})^{2}+(\mathrm{rd} \phi)^{2}=(\operatorname{Rd} \theta)^{2}+(\operatorname{rd} \phi)^{2}$

We know that

$\mathrm{r}=\mathrm{R} \sin (\theta)$

Differentiating equation, we get

$\mathrm{dr}=\mathrm{R} \cos (\theta) \mathrm{d} \theta$

Rearranging the terms, we get

$\operatorname{Rd} \theta=\frac{d r}{\cos \theta}=\frac{R d r}{\sqrt{\left\{R^{2}-r^{2}\right\}}}=\frac{R d r}{\sqrt{1-\frac{r^{2}}{R^{2}}}}$

The equation is expressed in terms of the plane polar coordinates $\mathrm{r}$ and $\varphi$.In general the curvature $\mathrm{K}$ of a twodimensional surface

$(\mathrm{dl})^{2}=\left(\frac{\mathrm{dr}^{2}}{\sqrt{\left\{1-\mathrm{Kr}^{2}\right\}}}\right)^{2}+(\mathrm{rd} \phi)^{2}$

Extending this to three dimensions by changing from polar to spherical coordinates we get

$(\mathrm{dl})^{2}=\left(\frac{\mathrm{dr}{ }^{2}}{\sqrt{\left\{1-\mathrm{Kr}^{2}\right\}}}\right)^{2}+(\mathrm{rd} \theta)^{2}+(\mathrm{r} \sin \theta \mathrm{d} \phi)^{2}$

where $r$ is the radial coordinate.

This equation shows the effect of curvature on spatial distances of a three-dimensional Universe. Next, we proceed to extend this by adding time to the space time metric. We measure both the points of observation at the same time from the fundamental principles of cosmology which implies that the universe is homogenous and isotropic in nature time cannot pass at different measures at different places. Hence, we keep this cdt 2 as a temporary quantity. Then the final equation of the metric is reduced to $(\mathrm{ds})^{2}=(\mathrm{cdt})^{2}-\left(\frac{\mathrm{dr}^{2}}{\sqrt{\left\{1-\mathrm{kr}^{2}\right\}}}\right)^{2}-(\mathrm{rd} \theta)^{2}-(\mathrm{r} \sin \theta \phi)^{2}$

We can now change the radial quantity to a moving quantity

$r(t)=a(t) \cdot x$

We define time dependent curvature on the basis of the scale factor and time dependent constant $\mathrm{k}$

$\mathrm{K}(\mathrm{t})=\frac{\mathrm{k}}{\mathrm{a}^{2}(\mathrm{t})}$

Finally, we substitute the values of $\mathrm{r}$ and $\mathrm{K}$ arriving at the Robertson-Walker Metric is given by $(\mathrm{ds})^{2}=(\mathrm{cdt})^{2}-$ $\mathrm{a}(\mathrm{t})^{2}\left[\left(\frac{\mathrm{dr}}{\sqrt{1-\mathrm{kr}^{2}}}\right)^{2}+\mathrm{r}^{2}\left(\mathrm{~d} \theta^{2} \sin ^{2} \theta \mathrm{d} \phi^{2}\right)\right]$

where in $\mathrm{r}$ is now denoting the radial distance.

\subsection{Friedman Equation}

The Friedman Equation explains how the expansion of space varies with time. Instead of using the general relativity of Einstein which comprises of 16 non-linear ordinary differential equations. We prefer using Newtonian Physics to avoid complexities to arrive at the final equation.

Proof:

We start by considering the law of conservation of energy

$\mathrm{U}=\frac{1}{2} \mathrm{mv} \mathrm{v}^{2}+$ Potential Energy

$\mathrm{E}=\frac{-\mathrm{GMm}}{\mathrm{r}}$

where $M=\frac{4}{3} \pi r^{3} \rho$

We obtain the total energy by using substitution of equation (14)

$\mathrm{u}=\frac{1 \mathrm{mr} \mathrm{r}^{2}}{2}-\frac{4}{3} \pi \mathrm{G} \rho \mathrm{r}^{2} \mathrm{~m}$

Multiplying both sides with $\frac{2}{\mathrm{ma}^{2} \mathrm{x}^{2}}$ and rearranging we obtain

$\left\{\frac{\dot{a}}{\mathrm{a}}\right\}^{2}=\frac{8 \pi \mathrm{G} \rho}{3}-\frac{\mathrm{kc}}{\mathrm{a}^{2}}$

where $\mathrm{k}=\frac{-2 \mathrm{u}}{\mathrm{mc}^{2 \mathrm{x}^{2}}}$

We obtain the final Friedman Equation as

$\left\{\frac{\dot{\mathrm{a}}}{\mathrm{a}}\right\}^{2}=\frac{8 \pi \mathrm{G} \rho}{3}-\frac{\mathrm{kc}}{\mathrm{a}^{2}}$

Further we also obtain

$2 \frac{d \dot{a}}{a}+\left\{\frac{\dot{a}}{a}\right\}^{2}=\frac{-8 \pi G \rho}{c^{2}}-\frac{k c^{2}}{a^{2}}$

where $\rho$ is mass density and $p$ is pressure.

The first Friedmann equation (21) shows that the rate of cosmic expansion, $\dot{a}$, increases with the mass density $\rho$ of the universe. Subtracting it from the second Friedmann equation (22) we obtain the acceleration equation

$2 \frac{\mathrm{d} \dot{\mathrm{a}}}{\mathrm{a}}=\frac{-4 \pi \mathrm{G}\left(\rho \mathrm{C}^{2}+3 \rho\right)}{3 \mathrm{c}^{2}}-\frac{k c^{2}}{\mathrm{a}^{2}}$

This implies that the acceleration decreases with the increasing pressure and mass density, thereby obtaining the Freidman Equation

$\mathrm{H}_{0}^{2}=\frac{8 \pi \mathrm{G} \rho_{0}}{3}-\frac{\mathrm{kc}}{\mathrm{a}^{2}}$

We can conclude that the Hubble's parameter depends on density and curvature of the universe. From the above 
equation the critical density can be calculated if Hubble's parameter is assumed to be $70 \mathrm{~km} / \mathrm{s}$ megaparsec we end up with $9 \times 10^{-27} \mathrm{~kg} / \mathrm{m}^{3}$, which is very small compared to the Earth's $5500 \mathrm{~kg} / \mathrm{m}^{3}$.

\section{STOCHASTIC DIFFERENTIAL EQUATION}

\subsection{Ornstein-Uhlenbeck process} Proof:

We obtain the following differential equation by comparison from eq (24) and (21)

$\mathrm{a}(\mathrm{t})=\mathrm{a}(\mathrm{t}) \mathrm{H}_{0}$

We introduce the stochastic differential equation by rewriting it as follows.

A(t) $\mathrm{f}\left(\mathrm{t}, \mathrm{A}_{\mathrm{t}}\right)+\mathrm{F}\left(\mathrm{t}, \mathrm{A}_{\mathrm{t}}\right) \in \mathrm{t}, \forall \mathrm{t}>0$

given the initial condition

$\mathrm{A}_{0}=\mathrm{a}_{0} \forall \mathrm{a}_{0} \in \mathrm{R}$

We now obtain it in the form of Ornstein-Uhlenbeck process

$\dot{A(t)}=u A_{t} d t+v A_{t} d W_{t}$

Lemma:

[2],[3],[4] Let $\mathrm{f}$ be a bounded Borel function on $\mathrm{R} 2, \tau$ is the stopping time with respect to $\mathrm{F}_{\tau}^{\mathrm{m}}, \tau<\propto$ with probability 1 .

Then,

$\mathrm{E}^{\mathrm{x}}\left[\mathrm{f}\left(\mathrm{X}_{\tau+\mathrm{h}} \mid \mathrm{F}_{\tau}^{\mathrm{m}}\right]=\mathrm{E}_{\tau}^{\mathrm{X}}\left[\mathrm{f}\left(\mathrm{X}_{\mathrm{h}}\right)\right] \forall \mathrm{h} \geq 0\right.$

Using the Itô Integral.

$A(t)=A(0)+\int_{0}^{t} u\left(s, A_{s}\right) u+\int_{0}^{t} v\left(s, A_{s}\right) d W_{s}$

The terms $\mathrm{u}(\mathrm{s}, \mathrm{As})$ and $\mathrm{v}(\mathrm{s}, \mathrm{Aa}) \mathrm{dWs}$ are the drift and the diffusion terms, due to the presence the white noise it becomes a stochastic differential equation

\section{ANALYTICAL METHOD FOR SOLVING THE STOCHASTIC DIFFERENTIAL EQUATION}

\subsection{Euler Maruyama Method for Simulation}

The most important part is to deal with $\delta \mathrm{W}_{\mathrm{i}}$ so here we assume that every observation $z_{i}$ will be a standard normal variable $N(0,1)$ with mean zero and variance to be one. Each value in the sample set $\mathrm{w}_{\mathrm{i}}=\left\{\mathrm{w}_{0}, \mathrm{w}_{1} \ldots ..\right\}$ produced by the Euler Maruyama method is an approximated realization of the solution. What we are basically trying to do is retrieve the expected values of function $\mathrm{f}$ or in general the path-dependent functions of the solution. But since the Wiener Process is not differentiable, we try to obtain the solution by integrating on infinitesimally smaller time scales using the Itô Integral

$a_{n+1}=a_{n}+\delta f\left(a_{n}, t_{n}\right)$

By using the Euler recursive method, we can solve the system of equations by using simulation techniques.

\subsection{Chapman Kolmogorov Forward Differential Equation}

For manual method we introduce the the factor eut and look at what happens to the stochastic process At. We get the following equations

$$
\begin{aligned}
& d\left(e^{u t} A_{t}\right)=e^{u t} d A_{t}+A_{t} d\left(e^{u t}\right) \\
& =e^{u t}-u d A_{t} d t+v d W_{t}+u A_{t} e^{u t} d t \\
& =v e^{u t} d W_{t}
\end{aligned}
$$

Integrating from 0 to $t$, we use integration by parts of the Ito integral

$$
\begin{aligned}
& =e^{u t} A_{t}=a_{0}+\int_{0}^{t} v e^{u t} d W_{s} \\
& =A_{t}=a_{0} e^{-u t}+v \int_{0}^{t} e^{\{u(s-t)\}} d W_{s}
\end{aligned}
$$

Theorem:

[5],[6],[7],[8] let $\mathrm{p}(\mathrm{a}, \mathrm{k}, \mathrm{Ht})$ be defined when $\mathrm{p}$ satisfies the forward Chapman Kolmogorov differential equations:

$=\frac{\partial \mathrm{p}(\mathrm{a}, \mathrm{k}, \mathrm{Ht})}{\partial \mathrm{t}}=\mathrm{up}(\mathrm{a}, \mathrm{k}, \mathrm{Ht})$

$\lim \mathrm{p}(\mathrm{a}, \mathrm{k}, \mathrm{Ht})=\delta_{\mathrm{a}}$

the operator $u$ is defined by

uf $=\sum_{\mathrm{i}} \frac{\mathrm{u}_{\mathrm{i}} \partial \mathrm{f}}{\partial \mathrm{x}_{\mathrm{i}}}+\frac{1}{2} \sum_{\mathrm{ij}} \mathrm{v}_{\mathrm{ij}}\left\{\frac{\partial^{2} \mathrm{f}}{\partial \mathrm{a}_{\mathrm{i}} \partial \mathrm{a}_{\mathrm{j}}}\right\}$

Where $\mathrm{v}_{\mathrm{ij}}=\sum_{\mathrm{jk}} \mathrm{v}_{\mathrm{ik}}(\mathrm{t}, \mathrm{a}) \mathrm{v}_{\mathrm{jk}}(\mathrm{a})$

Proposition:

$=A_{t}=a_{0} e^{-u t}+v\left(W_{t}-u \int_{0}^{t} e^{u(s-t)} W_{s} d s\right.$

Now we can obtain the pdf for the solution of the stochastic differential equation by using the [9],[10],[12],[16] ChapmanKolmogorov forward differential equation. The stochastic differential equation for a time-inhomogeneous case has the form.

$\frac{d a_{i}}{d t}=u_{i}(A, t)+v_{i k} \sum_{k} a, t \epsilon_{k}(t)$

Using the Kramers-Moyal Expansion we obtain the following equation.

$\frac{\partial \mathrm{P}}{\partial \mathrm{t}}=\sum_{\mathrm{i}=1} \mathrm{k} \frac{\partial}{\partial \mathrm{a}_{\mathrm{i}}}\left[\mathrm{u}_{\mathrm{i}}(\mathrm{a}, \mathrm{t})+\mathrm{D} \sum_{\mathrm{jk}} \mathrm{v}_{\mathrm{jk}}(\mathrm{a}, \mathrm{t}) \frac{\partial \mathrm{v}_{\mathrm{ik}}(\mathrm{a}, \mathrm{t})}{\partial \mathrm{a}_{\mathrm{j}}}\right] \mathrm{P}$

Proof:

This is entirely determined by the terms in the OrnsteinHollenbeck process. Using the above equations, we can use the forward Kolmogorov equation for the OrnsteinUhlenbleck process which is given below.

$\frac{\partial \mathrm{p}}{\partial \mathrm{t}}=\mathrm{u} \frac{\partial}{\partial \mathrm{a}}(\mathrm{ap})+\frac{1}{2} \mathrm{v}^{2} \frac{\partial^{2} \mathrm{p}}{\partial \mathrm{a}^{2}}$

where $\mathrm{p}(0, \mathrm{a})=\delta\left(\mathrm{a}-\mathrm{a}_{0}\right)$

We can now use Fourier transformation and get

$\frac{\partial \hat{p}}{\partial t}+u H \frac{\partial \widehat{p}}{\partial H}=\frac{-1}{2} v^{2} H^{2} \hat{p}$

We now use the method of characteristics to obtain the following equations.

$\frac{\partial \widehat{p}}{\partial \mathrm{t}}=\frac{\partial \widehat{\mathrm{p}}}{\partial \mathrm{t}}+\frac{\partial \widehat{\mathrm{p} d H}}{\partial \mathrm{Hdt}}=\partial \hat{\mathrm{p}} \partial \mathrm{t}+\mathrm{uH} \frac{\partial \widehat{\mathrm{p}}}{\partial \mathrm{H}}$

$\mathrm{H}=\mathrm{H}(0) \mathrm{e}^{\mathrm{ut}}$

$\frac{\partial \hat{p}}{\partial t}=\frac{-1}{2} v^{2} H^{2} \hat{p}$

$\frac{\partial \mathrm{H}}{\partial \mathrm{t}}=\mathrm{uH}$

$=\frac{-1}{2} H(0)^{2} e^{2 u t} v^{2} \hat{p}$ 
We know that

$\hat{\mathrm{p}}(0, \mathrm{H})=\mathrm{e}^{-\mathrm{iHa}_{0}}$

We obtain the following equation

$\hat{\mathrm{p}}(\mathrm{t}, \mathrm{H})=\hat{\mathrm{p}}(0, \mathrm{H}(0)) \mathrm{e}^{\left\{-\int_{0}^{\mathrm{t} 1} \mathrm{v}^{2} \mathrm{H}(0)^{2} \mathrm{e}^{2 \mathrm{us}} \mathrm{ds}\right\}}$

$=\mathrm{e}^{\left\{-\mathrm{iHa} \mathrm{a}_{0} \mathrm{e}^{-\mathrm{ut}}\right\}}-\frac{1 \mathrm{v}^{2}}{4 \mathrm{u}} \mathrm{e}^{-2 \mathrm{ut}\left(\mathrm{e}^{2 \mathrm{ut}}-1\right)}$

We can see from the equation obtained to be the same form as the Fourier transform of the probability density function with mean $\mathrm{a}_{0} \mathrm{e}_{\mathrm{ut}}$ and variance as $\frac{1}{2 \mathrm{u}} \mathrm{e}^{2 \mathrm{ut}}-1$

We obtain the pdf of the Fourier inverse as follows

$p(t, a)=\frac{1}{\sqrt{2 \pi v \frac{v^{2}}{2 u} e^{-2 u t}\left(e^{2 u t}-1\right)}} e^{\frac{-\left(a-a_{0} e^{-u t}\right)^{2}}{u}}$

This implies that using the time-inhomogeneous stochastic equations of Ornstein-Uhlenbeck process we can propose that the all the dynamical process that has had some effect on the evolution may be due to the scale-invariant timeinhomogeneous distribution of the galaxies .This proves that the changes in the Hubble's parameter varies stochastically with time. The impasse in the observation of the Hubble's parameter which has been a major debate worldwide in terms of the conjectural value of the Hubble's parameter can therefore be argued that the expansion rate of the Universe varies stochastically leading to the randomness in the recession velocities of the galaxies.

\section{RESULT ANALYSIS}

\subsection{Sensitivity Analysis}

\begin{tabular}{rrr|rrr}
\hline galaxy & velocity & distance & galaxy & velocity & distance \\
\hline NGC0300 & 133 & 2.00 & NGC3621 & 609 & 6.64 \\
NGC0925 & 664 & 9.16 & NGC4321 & 1433 & 15.21 \\
NGC1326A & 1794 & 16.14 & NGC4414 & 619 & 17.70 \\
NGC1365 & 1594 & 17.95 & NGC4496A & 1424 & 14.86 \\
NGC1425 & 1473 & 21.88 & NGC4548 & 1384 & 16.22 \\
NGC2403 & 278 & 3.22 & NGC4535 & 1444 & 15.78 \\
NGC2541 & 714 & 11.22 & NGC4536 & 1423 & 14.93 \\
NGC2090 & 882 & 11.75 & NGC4639 & 1403 & 21.98 \\
NGC3031 & 80 & 3.63 & NGC4725 & 1103 & 12.36 \\
NGC3198 & 772 & 13.80 & IC4182 & 318 & 4.49 \\
NGC3351 & 642 & 10.00 & NGC5253 & 232 & 3.15 \\
NGC3368 & 768 & 10.52 & NGC7331 & 999 & 14.72 \\
\hline
\end{tabular}

Source: From Freedman W. L., et al., The Astrophysical Journal, 553, 47-72,

Fig. 2: HUBBLE DATA

Call:

$\operatorname{lm}($ formula $=$ r\$velocity $*$ r\$distance $)$

Coefficients:

(Intercept) r\$distance

$6.696 \quad 76.127$

Fig. 3: HUBBLE'S LAW

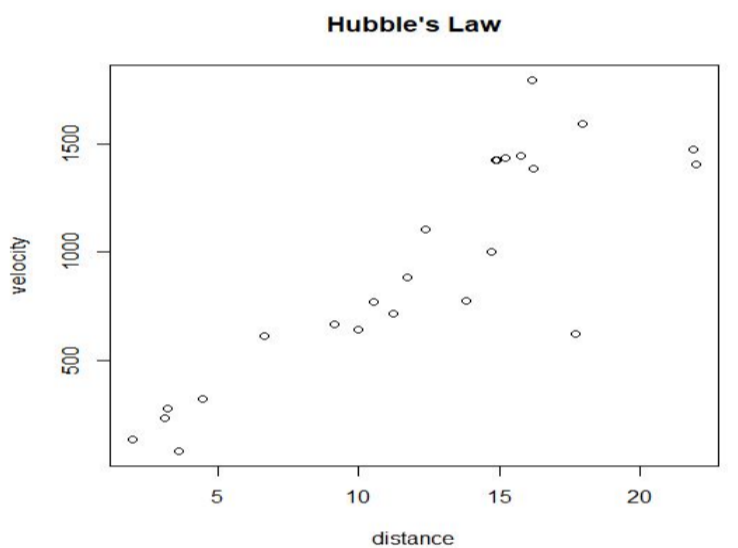

Fig. 4: HUBBLE's PARAMETER: GRAPHS
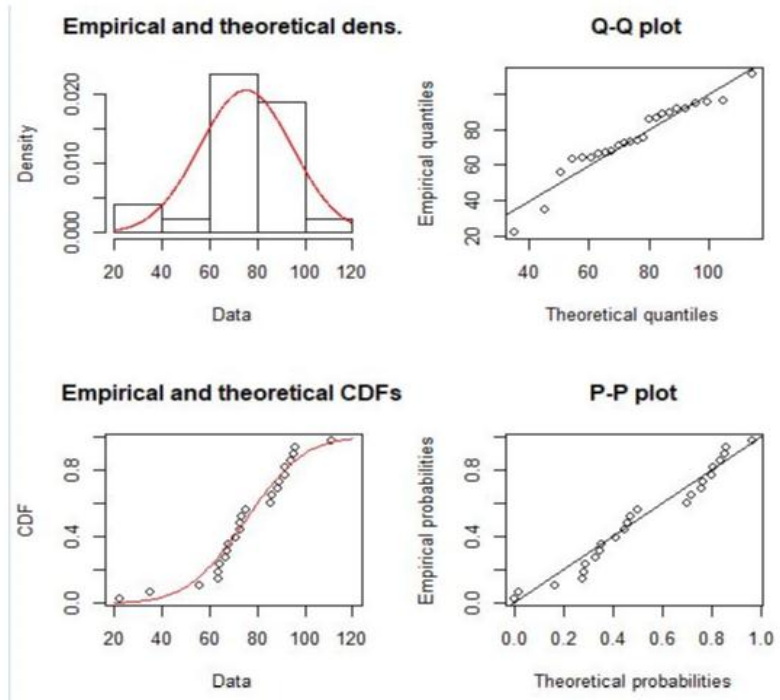

Fig. 4: HUBBLE's PARAMETER: GRAPHS

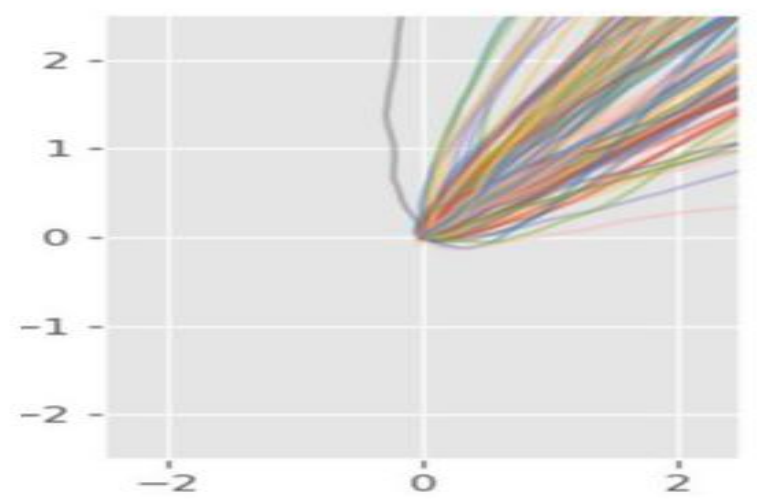

Fig. 5: SIMULATED GRAPHS OF THE HUBBLE'S PARAMETER

The Hubble data obtained from the observations of the Hubble Telescope published in the Astrophysics Journal gives the recessional velocities and distance of 24 galaxies where these distances are measured using the dependence between the period of Cepheid variable stars and their luminosity.[13],[14], [15] We can observe that the slope of the hobbles law using linear regression of the data with the help of the R-software is $76.127 \mathrm{~km} / \mathrm{sec}$ megaparsec which seems 
to be relatively constant as indicated by the graph of the Hubble's law. The reason why it is termed as a constant is because the Universe is expanding at the same measure everywhere in the Universe implying the Hubble's parameter is constant throughout space. But if we look through large distances and simulate it we can observe that the expansion rate no longer follows a straight line but is curved as seen by the different lines curved through moving out in the graph 2.This gives us valid proof for the hypothesis that the Hubble's parameter is neither a constant nor does it remains same everywhere in the Universe. These specific variations are due to the perturbations of the relativistic motion of individual galaxies through space causing this trend which changes for larger scales.

\subsection{Simulation Results}

Using the Python software to code the simulation of the stochastic nature of the Hubble's parameter these observations have been simulated based on the different instances of time as seen in the graphs below. The simulation algorithm follows the technique proposed above in the paper. We can observe how the observations simulated for different instances of time varies stochastically. From these graphs we observe that as the instance of time increases, the distance covered is also increasing and so is the volume of the space indicating the process of expansion at future time scale.
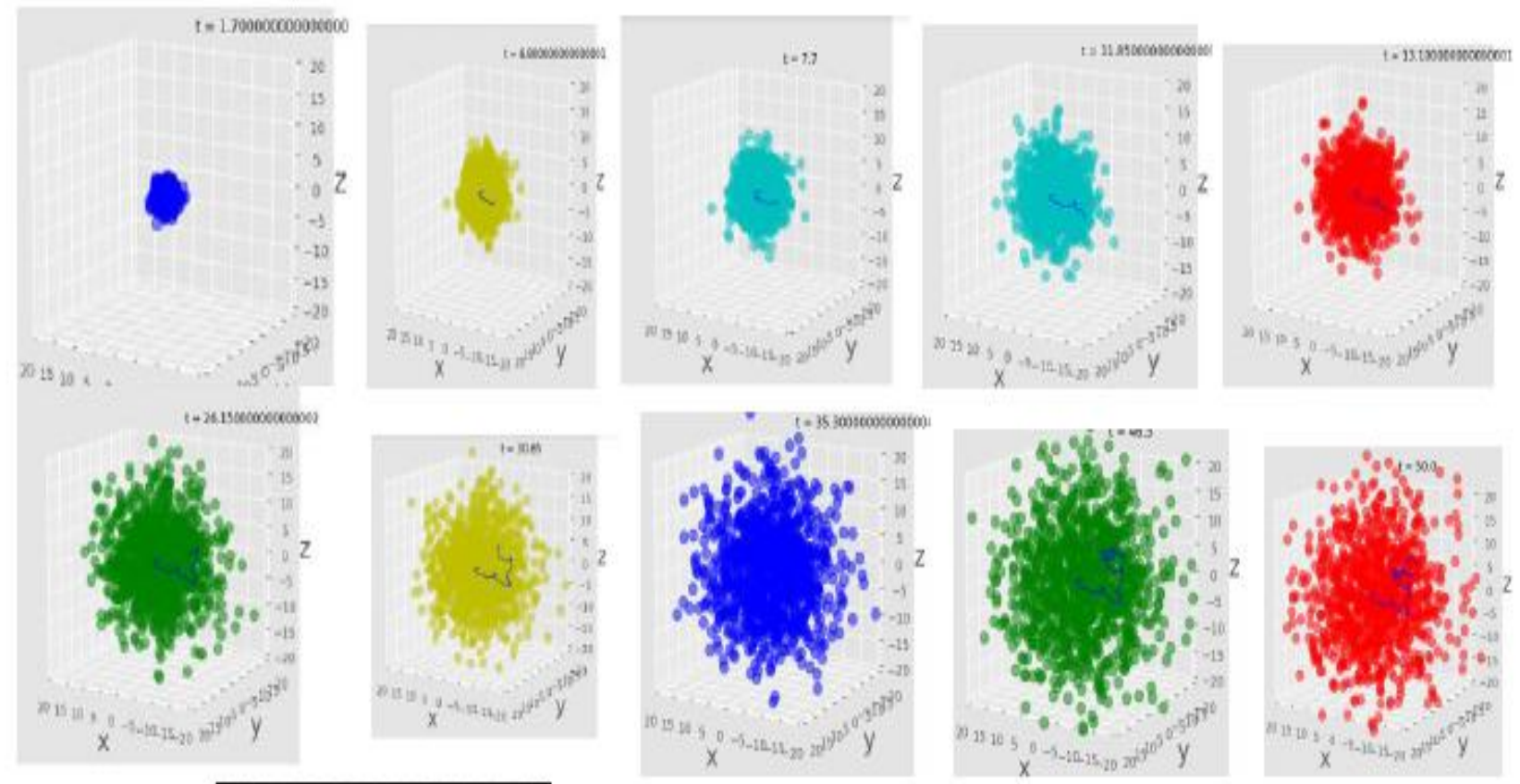

HUBBLE'S PARAMTER

Fig. 6: 3-D GRAPHS OF THE HUBBLE'S PARAMETER VARYING AT DIFFERENT INSTANCES

\section{CONCLUSION}

The proposal of this paper was to verify that the Hubble's parameter does not remain constant at large distances which has been validated using the R-software and the Python codes to simulate these observations for larger time scale. Conjectures regarding the component of the dark energy and dark matter for the expansion of the Universe has still not been confirmed or the presence of some other factors leading to this cause has still been speculated. From this paper we can majorly conclude the premise by this line of reasoning that due to random forces that we have considered while simulation has eventually led to the stochastic behaviour of the Hubble's parameter giving us major ideas that density may tend to also be varying stochastically with time leading to further research. Apart from these two conclusions we can reason out that since luminous distance is associated with Doppler's effect and thereby on the Hubble's parameter which can lead us to only propose this new hypothesis that the rate of the expansion of the Universe varies stochastically leading to scale-invariance.

\section{ACKNOWLEDGMENT}

The author would like to thank the reviewers for their constructive and insightful comments with respect to this paper. Also, a special note of gratitude is rendered to all the near and dear ones who supported immensely in the completion of this paper

\section{REFERENCES}

[1] Bagla J, Hubble's Law and expanding universe. Resonance, March 2009.

[2] Imran Masood, A two stage interval stochastic programming

[3] Mao. X, Stochastic Differential Equations and Applications,Eleviser 2007

[4] Oksendal B, Stochastic Differential Equation.

[5] Papoulis and Pillai, Probability Random Process and stochastic process

[6] Protter.PE, Stochastic Differential Equations, Stochastic Integration and Differential Equations, Springer 249361,2005

[7] MD Raisinghania Ordinary and Partial Differential Equations

[8] Samuel Karli Howard M Taylor, A first course in 
stochastic process, $3^{\text {rd }}$ Edition, Academic Press

[9] Shapiro Dentcheva Lectures on Stochastic Modelling

[10] Shapiro Phipott,A tutorial on stochastic programming,

[11] Sivakumar Stochastic evolution of the evolution of the Universe, Indian Academy of Sciences.

[12] Oliver Knill, Probability and Stochastic Processes with Applcations,2002, Overseas Press.

[13] Alexandre J.Chorin and Ole H Haldr, Stochastic Tools for Mathematica and Sciences,Berkeley,California
March,2009.

[14] Gustav Ludvigsson,Kolmogorov Equations,June 2003 Uppasala Universtat

[15] C.W Gardiner,Handbook of Stochastic Methods for Physics,Chemsitry and the Natural Sciences, $2^{\text {nd }}$ Edition,Springer

[16] Rabi N Battavharya Edward C Wayemire,Stochastic Processes with Applications,SIAM.

[17] Waset abstract selected for ICABS 2020 